\title{
WAVE FUNCTION OF THE HARMONIC OSCILLATOR IN CLASSICAL STATISTICAL MECHANICS
}

\author{
Vladimir N. Chernega ${ }^{1}$ and Vladimir I. Man'ko \\ ${ }^{1}$ Faculty of Physics, M.V. Lomonosov Moscow State University \\ Vorob'evy Gory, Moscow 119992, Russia \\ ${ }^{2}$ P.N. Lebedev Physical Institute, Russian Academy of Sciences \\ Leninskii Prospect, 53, Moscow 119991, Russia \\ e-mails: vchernega@gmail.com manko@sci.lebedev.ru
}

\begin{abstract}
The notion of wave function of the classical harmonic oscillator is discussed. The evolution equation for this wave function is obtained using the classical Liouville equation for the probability-distribution function of the harmonic oscillator. The tomographic-probability distribution of the classical oscillator is studied. Examples of the ground-like state and the coherent state of the classical harmonic oscillator are considered.
\end{abstract}

Keywords: classical mechanics, harmonic oscillator, probability, wave function, tomography, coherent state, Radon transform.

\section{Introduction}

The states of the oscillator in classical mechanics are described by the position $q$ and momentum $p$. If one has fluctuations of the position and momentum, the states of such a classical oscillator are described by the probability-distribution density $f(q, p)$, which is a nonnegative normalized function on the oscillator phase space $[1,2]$. The states of the quantum oscillator are described by the wave function (pure states) or the density matrix (mixed states); see, e.g., [3]. Recently it was shown that the states of quantum systems can be described by the probability-distribution function called tomogram [4]. This means that the states of the quantum system and the states of the classical system can be described by the same method using the probability distribution for both quantum and classical systems [5,6]. But this similarity of describing the classical and quantum systems provides an idea of introducing a wave function (or density matrix) for the description of states of classical systems like in quantum mechanics. Some aspects of this idea were discussed in recent publications $[7,8]$.

The aim of our work is to study in detail a classical harmonic oscillator by introducing for its state the wave function and density matrix. In the paper, we construct the creation and annihilation operators for the classical harmonic oscillator, discuss the Hilbert space associated to the states of the classical oscillator, and construct the analogs of excited and coherent states of the classical oscillator.

The paper is organized as follows.

In Sec. 2 a review of classical phase-space properties is presented. In Sec. 3 the integral Radon transform is discussed. In Sec. 4 the wave function determined by a probability distribution is considered.

Manuscript submitted by the authors in English on August 20, 2007.

1573-8760/07/2806-0535 두2007 Springer Science+Business Media, Inc. 
In Sec. 5 examples of the ground and excited states of the classical oscillator are shown. In Sec. 6 the evolution equation for the wave function of the classical oscillator is derived. In Sec. 7 the Gaussian solution of the evolution equation are studied. In Sec. 8 the Hilbert space is associated to the classicaloscillator states. In Sec. 9 the conclusions and perspectives are given.

\section{Rotations in the Phase Space and Analogy with Lorentz Transform}

In order to introduce the notion of wave function for the classical harmonic oscillator, let us study rotations in its phase space. The Hamiltonian of the classical harmonic oscillator reads

$$
H=\frac{p^{2}}{2}+\frac{q^{2}}{2}
$$

(we take the frequency and mass $\omega=m=1$ ). The equations of motion

$$
\frac{\partial H}{\partial p}=\dot{q}, \quad-\frac{\partial H}{\partial q}=\dot{p}
$$

provide the standard differential equations

$$
p=\dot{q}, \quad-q=\dot{p},
$$

that yields

$$
\ddot{q}+q=0 .
$$

The integrals of motion $I(q, p, t)$ satisfy the equation for the total derivative of this function

$$
\frac{d}{d t} I(q, p, t)=0
$$

The total derivative reads

$$
\frac{d}{d t} I(q, p, t)=\frac{\partial I(q, p, t)}{\partial t}+\frac{\partial I(q, p, t)}{\partial q} \dot{q}+\frac{\partial I(q, p, t)}{\partial p} \dot{p} .
$$

This means that any integral of motion of the classical harmonic oscillator satisfies the partial differential equation [9]

$$
\frac{\partial I(q, p, t)}{\partial t}+p \frac{\partial I(q, p, t)}{\partial q}-q \frac{\partial I(q, p, t)}{\partial p}=0 .
$$

If a harmonic oscillator interacts with a medium, the position and momentum of the oscillator fluctuate. This means that the state of the classical harmonic oscillator is described by a probability distribution function $f(q, p, t)$ in the phase space. This function is nonnegative

$$
f(q, p, t) \geq 0
$$

and satisfies the normalization condition

$$
\int f(q, p, t) d q d p=1
$$


which means that the total probability of finding the oscillator on the phase space is equal to unity. The probability distribution satisfies the Liouville kinetic equation for the oscillator

$$
\frac{\partial f(q, p, t)}{\partial t}+p \frac{\partial f(q, p, t)}{\partial q}-q \frac{\partial f(q, p, t)}{\partial p}=0 .
$$

Equations (10) and (7) are identical. This means that the probability distribution $f(q, p, t)$ is the integral of motion for the harmonic oscillator.

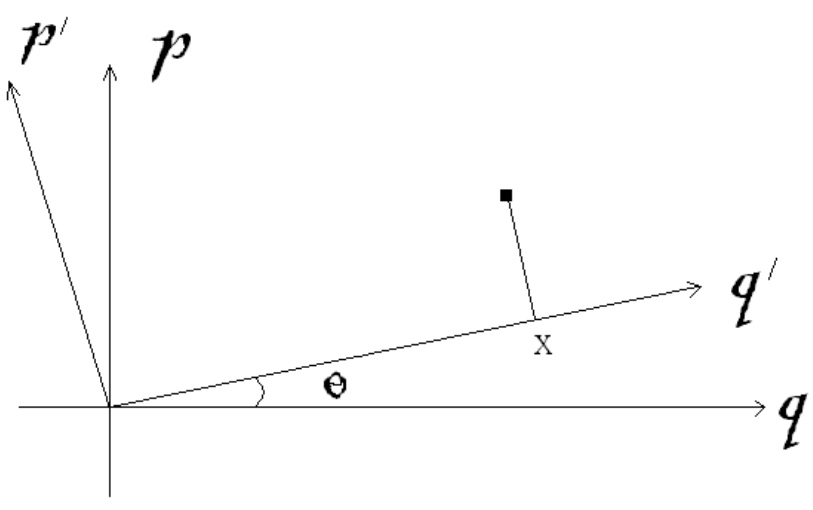

Fig. 1. The initial and rotated reference frames and the position of the oscillator in the rotated reference frame.

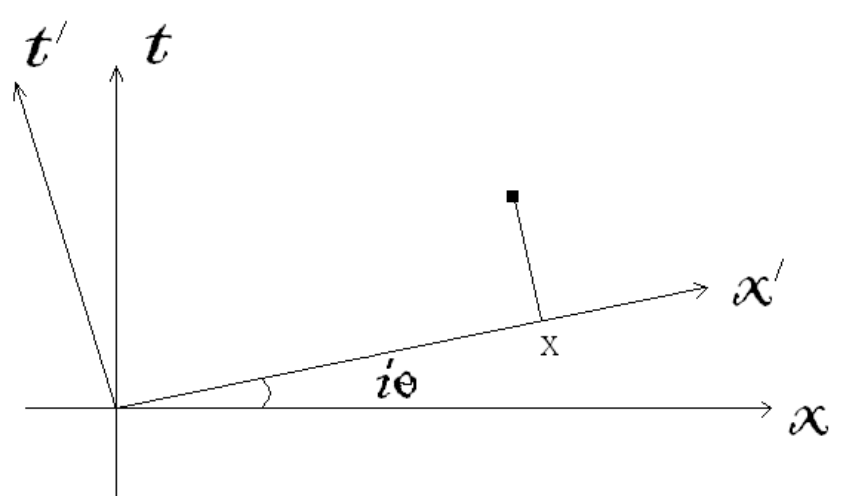

Fig. 2. The initial and hyperbolically rotated reference frames in the space-time plane of a relativistic particle corresponding to the Lorentz transform.

Let us make the rotation in the phase space. We introduce the coordinates

$$
X=q \cos \Theta+p \sin \Theta, \quad P=-q \sin \Theta+p \cos \Theta .
$$

The position $X$ is shown on the phase space in Fig. 1 where we introduced two reference frames, namely, the initial reference frame with axes $q$ and $p$ and rotated reference frame with axes $q^{\prime}$ and $p^{\prime}$. The rotated reference frame is an analog of the Lorentz transform of the position of a relativistic particle and time. In fact, for a moving reference frame with velocity $v$ along the $x$ axis of the initial reference frame, the position and time are expressed as follows:

$$
x=\frac{x^{\prime}+v t^{\prime}}{\sqrt{1-v^{2}}}, \quad t=\frac{t^{\prime}+v x^{\prime}}{\sqrt{1-v^{2}}}
$$

(we take the speed of light $c=1$.) If one introduces the angle $\Theta$ by the relations

$$
\cosh \Theta=\frac{1}{\sqrt{1-v^{2}}}, \quad \sinh \Theta=\frac{v}{\sqrt{1-v^{2}}},
$$

the Lorentz transform (12) becomes similar to rotation (11). The difference is that the angle $\Theta$ becomes purely imaginary $\Theta \rightarrow \imath \Theta$. The imaginary rotation of the space-time reference frame is shown in Fig. 2 . We know that the Lorentz transform explains all the unusual effects of special relativity. The rotations in the phase space can explain the unusual effects of quantum mechanics [4]. In this work, we focus on the role of the reference-frame rotation for the classical oscillator in its phase space. 


\section{Radon Transform}

In 1917 Radon introduced the integral transform of a function of two variables [10], nowadays known as the Radon transform. We write this transform for the probability distribution $f(q, p)$. For a while, we omit the time dependence of this function, e.g., considering the function at the time moment $t=0$. The transform reads

$$
w(X, \mu, \nu)=\int f(q, p) \delta(X-\mu q-\nu p) d q d p
$$

where

$$
\mu=\cos \Theta, \quad \nu=\sin \Theta .
$$

Since the delta-function is equal to zero everywhere excepting its zero argument, i.e.,

$$
X=q \cos \Theta+p \sin \Theta,
$$

one can see that the position $X$ of the oscillator in the rotated reference frame given by (11) is taken here. We used the notation $\mu$ and $\nu$ because, if before the rotation we make the scaling of initial axes (see Fig. 1) $q \rightarrow s q$ and $p \rightarrow s^{-1} p$ and then rotate the scaled axes of the reference frame, formula (14) is valid but the parameters $\mu$ and $\nu$ contain the scaling factor

$$
\mu=s \cos \Theta, \quad \nu=s^{-1} \sin \Theta .
$$

The Radon transform (14) is invertible, which means that the function $w(X, \mu, \nu)$ (called tomogram [4]) determines the probability distribution $f(q, p)$.

The inversion formula reads

$$
f(q, p)=\int w(X, \mu, \nu) e^{\iota(X-\mu q-\nu p)} \frac{d X d \mu d \nu}{(2 \pi)^{2}} .
$$

The properties of tomogram according to its construction are the properties of the marginal probability distribution.

\section{Wave Function Associated to the Probability Distribution}

In quantum mechanics, the state of the oscillator is described by the wave function $\Psi(x, t)$, which obeys the Schrödinger evolution equation. It reads

$$
\imath \frac{\partial \Psi(x, t)}{\partial t}=-\frac{1}{2} \frac{\partial^{2} \Psi(x, t)}{\partial x^{2}}+\frac{x^{2}}{2} \Psi(x, t) .
$$

We take the frequency $\omega=1$, mass $m=1$, and Planck's constant $\hbar=1$. In quantum mechanics, the density matrix $\rho\left(x, x^{\prime}, t\right)$, which is a function of two variables $x$ and $x^{\prime}$ and time $t$, is used to describe the pure and mixed quantum states. The pure quantum state has the wave function $\Psi(x, t)$ and the density matrix of this state reads

$$
\rho_{\Psi}\left(x, x^{\prime}, t\right)=\Psi(x, t) \Psi^{*}\left(x^{\prime}, t\right)
$$


For mixed states, the density matrix is a mixture of density matrices of pure states

$$
\rho\left(x, x^{\prime}, t\right)=\sum_{k} p_{k} \rho_{\Psi_{k}}\left(x, x^{\prime}, t\right)
$$

where $p_{k} \geq 0$ and $\sum_{k} p_{k}=1$.

In quantum mechanics, the Wigner function [11] is also used to describe the quantum state with the density matrix $\rho\left(x, x^{\prime}, t\right)$.

The Wigner function is given by the Fourier transform of the density matrix, i.e.,

$$
W(q, p, t)=\int \rho\left(q+\frac{u}{2}, q^{\prime}-\frac{u}{2}\right) e^{-\imath p u} d u .
$$

The inverse Fourier transform yields

$$
\rho\left(x, x^{\prime}, t\right)=\frac{1}{2 \pi} \int W\left(\frac{x+x^{\prime}}{2}, p, t\right) e^{\imath p\left(x-x^{\prime}\right)} d p .
$$

For pure states, these formulas have the form

$$
W_{\Psi}(q, p, t)=\int \Psi\left(q+\frac{u}{2}, t\right) \Psi^{*}\left(q-\frac{u}{2}, t\right) e^{-\imath p u} d u .
$$

The inverse transform gives the wave function in terms of the Wigner function $W_{\Psi}(q, p, t)$, i.e.,

$$
\Psi(x, t) \Psi^{*}\left(x^{\prime}, t\right)=\frac{1}{2 \pi} \int W_{\Psi}\left(\frac{x+x^{\prime}}{2}, p, t\right) e^{\imath p\left(x-x^{\prime}\right)} d p .
$$

The above formula yields

$$
\Psi(0, t) \Psi^{*}(0, t)=\frac{1}{2 \pi} \int W_{\Psi}(0, p, t) d p
$$

Also one has

$$
\Psi(x, t) \Psi^{*}(0, t)=\frac{1}{2 \pi} \int W_{\Psi}\left(\frac{x}{2}, p, t\right) e^{\imath p x} d p
$$

or

$$
\Psi(x, t)=\frac{1}{2 \pi \Psi^{*}(0, t)} \int W_{\Psi}\left(\frac{x}{2}, p, t\right) e^{\imath p x} d p
$$

Since

$$
\Psi(0, t)^{*}=\left|\Psi^{*}(0, t)\right| e^{-\imath \varphi_{0}(t)},
$$

where $\varphi_{0}(t)$ is the time-dependent phase, one has

$$
\Psi(x, t)=\frac{1}{\sqrt{2 \pi}} \frac{e^{\imath \varphi_{0}(t)}}{\sqrt{\int W_{\Psi}(0, p, t) d p}} \int W_{\Psi}\left(\frac{x}{2}, p, t\right) e^{\imath p x} d p .
$$


The Wigner function of the pure state has the properties

$$
\int W(q, p, t) \frac{d p}{2 \pi}=|\Psi(q, t)|^{2}
$$

and

$$
\int W(q, p, t) \frac{d q}{2 \pi}=|\tilde{\Psi}(p, t)|^{2}
$$

The function $\widetilde{\Psi}(p, t)$ is the oscillator wave function in the momentum representation. These properties are analogous to the properties of probability distributions $f(q, p, t)$ of the classical oscillator. In view of this, one can introduce the density matrix and wave function of the classical harmonic oscillator using the following definition.

In formulas $(23)$ and $(30)$, we replace $W(q, p, t)$ by $2 \pi f(q, p, t)$. Thus one has the wave function of the classical harmonic oscillator

$$
\Psi_{f}(x, t)=\frac{1}{\sqrt{\int f(0, p, t) d p}} \int f\left(\frac{x}{2}, p, t\right) e^{\imath p x} d p .
$$

We take the phase factor $e^{\iota \varphi_{0}(t)}$ to be equal to 1 . The density matrix of the state of the classical harmonic oscillator reads

$$
\rho_{f}\left(x, x^{\prime}, t\right)=\int f\left(\frac{x+x^{\prime}}{2}, p, t\right) e^{\imath p\left(x-x^{\prime}\right)} d p
$$

\section{Ground and Excited States of the Classical Harmonic Oscillator}

Let us take the classical wave function of the harmonic oscillator in the form of the wave function of the ground state of the quantum oscillator [12], i.e.,

$$
\Psi_{\text {ocl }}(x)=\frac{1}{\sqrt[4]{\pi}} e^{-x^{2} / 2}
$$

where we take time $t=0$.

We introduce the creation and annihilation operators of the classical harmonic oscillator in the form used for the quantum oscillator. The annihilation operator reads

$$
\hat{a}=\frac{1}{\sqrt{2}}\left(x+\frac{\partial}{\partial x}\right)
$$

and the creation operator is

$$
\hat{a}^{\dagger}=\frac{1}{\sqrt{2}}\left(x-\frac{\partial}{\partial x}\right)
$$

The commutation relation of the creation and annihilation operators reads

$$
\left[\hat{a}, \hat{a}^{\dagger}\right]=\hat{a} \hat{a}^{\dagger}-\hat{a}^{\dagger} \hat{a}=\hat{1}
$$


where $\hat{1}$ is the identity operator. The identity operator is defined by the relation

$$
\hat{1} \Psi(x)=\Psi(x),
$$

where $\Psi(x)$ is an arbitrary function. One has the equality

$$
\hat{a} \Psi_{\text {ocl }}(x)=0
$$

Also, analogously to the quantum oscillator, we introduce the wave functions of the excited states

$$
\Psi_{n}^{\mathrm{cl}}(x)=\frac{\hat{a}^{\dagger n}}{\sqrt{n !}} \Psi_{\mathrm{ocl}}(x), \quad n=0,1,2, \ldots
$$

These functions are known and expressed in terms of Hermite polynomials

$$
\Psi_{n}^{\mathrm{cl}}(x)=\frac{e^{-x^{2} / 2}}{\sqrt[4]{\pi}} \frac{1}{\sqrt{2^{n} n !}} H_{n}(x) .
$$

The Hermite polynomial is determined by the generating function, i.e.,

$$
e^{-\tau^{2}+2 \tau x}=\sum_{n=0}^{\infty} \frac{\tau^{n}}{n !} H_{n}(x) .
$$

The classical distribution function of state (35) has the form

$$
f_{0}(q, p)=\frac{1}{\pi} e^{-q^{2}-p^{2}}
$$

Thus the distribution function (44) is the Gaussian joint probability distribution of two random variables which are the position and momentum. The mean values of the position and momentum are equal to zero, and the dispersions read

$$
\left(\sigma_{q}\right)^{2}=1 / 2, \quad\left(\sigma_{p}\right)^{2}=1 / 2 .
$$

One can check that the oscillator wave function (33) is related to the probability-distribution density $f(x, p, t)$ by the formula

$$
\int f(q, p, t) d p=\left|\Psi_{\mathrm{cl}}(q, t)\right|^{2}
$$

The meaning of the formal wave function of the classical oscillator is the same as in quantum mechanics. For its modulus, one has

$$
\left|\Psi_{\mathrm{cl}}(x, t)\right|^{2}=P(x, t)
$$

where $P(x, t)$ is the probability density of the oscillator position. 


\section{Evolution Equation for the Wave Function of the Classical Harmonic Oscillator}

One can introduce the evolution equation for the wave function of the harmonic oscillator using (10). In fact, if the wave function obeys the Schrödinger-like equation

$$
\iota \frac{\partial \Psi_{\mathrm{cl}}(x, t)}{\partial t}=-\frac{1}{2} \frac{\partial^{2} \Psi_{\mathrm{cl}}(x, t)}{\partial x^{2}}+\frac{1}{2} x^{2} \Psi_{\mathrm{cl}}(x, t)
$$

the function

$$
f(q, p, t)=\frac{1}{2 \pi} \int \Psi_{\mathrm{cl}}\left(q+\frac{u}{2}, t\right) \Psi_{\mathrm{cl}}^{*}\left(q-\frac{u}{2}, t\right) e^{-\iota p u} d u
$$

can be checked to satisfy Eq. (10). Thus we can consider (47) as a mathematical tool to solve (10). The tool consists of using the integral transforms (48) and (33). The phase factor in (33) was taken to be equal to 1 in comparison with (30). This means that we derive Eq. (10) for the oscillator starting from (47). If we want to derive Eq. (47) from (10), we need to introduce the phase factor as an additional information. One can pose the question - how it is possible to introduce the Schrödinger-like equation for the generic potential $U(q)$ ? As was shown in [7], it is possible to obtain only the von Neuman-like equation for the density matrix starting from (10). This means that the solutions of this equation do not have the form (20). The von Neuman equation in quantum mechanics has the form

$$
\iota \frac{\partial \rho\left(x, x^{\prime}, t\right)}{\partial t}=-\frac{1}{2} \frac{\partial^{2} \rho\left(x, x^{\prime}, t\right)}{\partial x^{2}}+U(x) \rho\left(x, x^{\prime}, t\right)+\frac{1}{2} \frac{\partial^{2} \rho\left(x, x^{\prime}, t\right)}{\partial x^{\prime 2}}-U\left(x^{\prime}\right) \rho\left(x, x^{\prime}, t\right) .
$$

This equation has solutions (pure states) of the form (20). Also this equation has solutions (mixed states) of the form (21). The equation for the classical density matrix (34), which one can obtain from (10), reads

$$
\iota \frac{\partial \rho_{\mathrm{cl}}\left(x, x^{\prime}, t\right)}{\partial t}=-\frac{1}{2} \frac{\partial^{2} \rho_{\mathrm{cl}}\left(x, x^{\prime}, t\right)}{\partial x^{2}}+\frac{1}{2} \frac{\partial^{2} \rho_{\mathrm{cl}}\left(x, x^{\prime}, t\right)}{\partial x^{\prime 2}}-\left(x^{\prime}-x\right) U^{\prime}\left(\frac{x+x^{\prime}}{2}\right) \rho_{\mathrm{cl}}\left(x, x^{\prime}, t\right)=0 .
$$

The difference between the two equations [(50) and (49)] is contained in terms with the potential energy. But for systems with potential energy of the quadratic form

$$
U(x)=a(t) x^{2}+b(t) x+c(t)
$$

both equations coincide. Due to this, (50) in the classical-oscillator case has solutions of the form of pure states

$$
\rho_{\mathrm{cl}}^{\Psi}\left(x, x^{\prime}, t\right)=\Psi_{\mathrm{cl}}(x, t) \Psi_{\mathrm{cl}}^{*}\left(x^{\prime}, t\right),
$$

which is preserved during the time evolution.

\section{Gaussian Solutions of the Schrödinger-like Equation for the Classical Oscillator}

One can check that specific Gaussian packets, i.e., wave functions of the form of the coherent state [9]

$$
\Psi_{\alpha}^{\mathrm{cl}}(x, t)=\pi^{-1 / 4} \exp \left(-\frac{x^{2}}{2}-\frac{\iota t}{2}\right) \exp \left(-\frac{|\alpha|^{2}}{2}+\sqrt{2} \alpha e^{-\iota t} x-\frac{\alpha^{2}}{2} e^{-2 \iota t}\right),
$$


satisfy the evolution equation for the classical oscillator. This function is the eigenfunction of the annihilation operator

$$
\hat{a} \Psi_{\alpha}(x, t)=\alpha e^{-\iota t} \Psi_{\alpha}(x, t),
$$

where $\alpha=\alpha_{1}+\iota \alpha_{2}$ is an arbitrary complex number.

One can check that $\Psi_{\alpha}(x, t)$ satisfies both Eq. (47) and the normalization condition

$$
\int \Psi_{\alpha}^{*}(x, t) \Psi_{\alpha}(x, t) d x=1 .
$$

The probability distributions $f(q, p, t)$ given by (48) in the coherent-state case has the form of the Gaussian joint probability density of random position and momentum

$$
f_{\alpha}(q, p, t)=\frac{1}{\pi} e^{-(p-\bar{p})^{2}-(q-\bar{q})^{2}}
$$

where

$$
\bar{p}=\sqrt{2} \operatorname{Im}\left(\alpha e^{-\iota t}\right), \quad \bar{q}=\sqrt{2} \operatorname{Re}\left(\alpha e^{-\iota t}\right) .
$$

The dispersions of the position and momentum in the coherent state of the classical oscillator read

$$
\left(\sigma_{p}\right)^{2}=\frac{1}{2}, \quad\left(\sigma_{q}\right)^{2}=\frac{1}{2} .
$$

There exist other Gaussian solutions such as squeezed states. Now we consider the solution of Eq. (47) in the form of the excited state of the quantum harmonic oscillator (41) and (42). In fact, the function

$$
\Psi_{n}^{c l}(x, t)=e^{-\iota(n+1 / 2) t} \frac{e^{-x^{2} / 2}}{\sqrt[4]{\pi}} \frac{1}{\sqrt{2^{n} n !}} H_{n}(x), \quad n=0,1,2 \ldots
$$

is the other solution of Eq. (47). For $t=0$, this function satisfies the relations

$$
\begin{aligned}
& \hat{a} \Psi_{n}^{\mathrm{cl}}(x, t)=\sqrt{n} \Psi_{n-1}^{\mathrm{cl}}(x, t), \\
& \hat{a}^{\dagger} \Psi_{n}^{\mathrm{cl}}(x, t)=\sqrt{n+1} \Psi_{n+1}^{\mathrm{cl}}(x, t),
\end{aligned}
$$

and orthogonality conditions

$$
\int \Psi_{n}^{\mathrm{cl} *}(x, t) \Psi_{m}^{\mathrm{cl}}(x, t) d x=\delta_{n m}
$$

analogously to the case of wave functions of the quantum oscillator. But if one calculates the probability $f_{n}(q, p, t)$ using formula (48), the function $f_{n}(q, p, t)$ will take both positive and negative values.

For example,

$$
f_{1}(q, p, t)=\frac{1}{2 \pi} \int \Psi_{1}^{\mathrm{cl}}\left(q+\frac{u}{2}, t\right) \Psi_{1}^{\mathrm{cl} *}\left(q-\frac{u}{2}, t\right) e^{-\iota p u} d u
$$

where

$$
\Psi_{1}^{c l}(x, t)=\sqrt{2} \pi^{-1 / 4} e^{-3 \iota t / 2} x e^{-x^{2} / 2}
$$


has the form

$$
f_{1}(q, p, t)=\frac{2}{\pi}\left(p^{2}+q^{2}-\frac{1}{2}\right) e^{-p^{2}-q^{2}}
$$

One can see that for small $p$ and $q$ (say, $p=0$ and $q=0$ ), the function is negative. This means that in classical mechanics this solution of the Schrödinger-like equation (48) is not admissible due to the physical meaning of probability distributions $f(q, p, t)$. This is an essential difference from the quantum oscillator. In quantum mechanics, such a function describes the state with the energy level $n=1$. The Wigner function $W_{1}(q, p, t)$ given by formula (24) for the quantum oscillator can take negative values.

\section{Hilbert Space of States for the Classical Oscillator}

Since we introduced the formalism of wave function for the classical oscillator, we can introduce the formalism of Hilbert space of states. In fact, considering the wave function using the Dirac notation

$$
\Psi(x)=\langle x \mid \Psi\rangle,
$$

we associate with the wave function $\Psi(x)$ the state vector $|\Psi\rangle$ belonging to the Hilbert space. The density matrix can also be considered using the notion of density operator $\hat{\rho}$ acting in the Hilbert space, i.e.,

$$
\Psi(x, t) \Psi^{*}\left(x^{\prime}, t\right)=\left\langle x\left|\hat{\rho}^{c l}(t)\right| x^{\prime}\right\rangle .
$$

In quantum mechanics, the density operator must be nonnegative. For the classical density operator, this property is violated.

Thus in classical mechanics of the harmonic oscillator we can formally introduce the structure which is used for the quantum harmonic oscillator. This structure contains the Hilbert space of the oscillator states, an analog of the Wigner function which is the probability-distribution density, and the Radon transform of this probability-distribution density, which we call tomogram of the oscillator state.

Now we consider the example of the ground state in terms of tomograms. The wave function is given by (35). The probability density is given by (44). The tomogram can be calculated using (14), and it reads

$$
w_{0}(X, \mu, \nu)=\frac{1}{\sqrt{\pi\left(\mu^{2}+\nu^{2}\right)}} e^{-X^{2} /\left(\mu^{2}+\nu^{2}\right)} .
$$

The tomogram is the Gaussian distribution with the mean value

$$
\langle X\rangle=0
$$

and dispersion

$$
\sigma_{X}^{2}=\frac{\mu^{2}+\nu^{2}}{2}
$$

The characteristic function of the Gaussian distribution has the form

$$
\varphi_{X}(t)=e^{-t^{2}\left(\mu^{2}+\nu^{2}\right) / 4} .
$$


Here $t$ is a parameter and

$$
\varphi_{X}(t)=\int w_{0}(X, \mu, \nu) e^{\imath t X} d X .
$$

The momenta of the probability-distribution density are determined by the series

$$
\begin{aligned}
\varphi_{X}(t) & =\int w_{0}(X, \mu, \nu)\left[1+\imath t X+\frac{(\imath t X)^{2}}{2 !}+\cdots+\frac{(\imath t X)^{n}}{n !}+\cdots\right] d X \\
& =1+\imath t\langle X\rangle+\frac{(\imath t)^{2}}{2 !}\left\langle X^{2}\right\rangle+\cdots+\frac{(\imath t)^{n}}{n !}\left\langle X^{n}\right\rangle+\cdots,
\end{aligned}
$$

where

$$
\left\langle X^{n}\right\rangle=\int w_{0}(X, \mu, \nu) X^{n} d X
$$

This means that we have the formula for the highest momentum in terms of the characteristic function

$$
\left\langle X^{n}\right\rangle=\left.\frac{d^{n}}{d(\imath t)^{n}} \varphi_{X}(t)\right|_{t=0} .
$$

For example, one has in (71)

$$
e^{-t^{2}\left(\mu^{2}+\nu^{2}\right) / 4}=1+\left(-\frac{1}{4}\right)\left(\mu^{2}+\nu^{2}\right) t^{2}+\left[-\frac{1}{4}\left(\mu^{2}+\nu^{2}\right)\right]^{2} \frac{t^{4}}{2 !}+\cdots
$$

One can see that for odd $n=2 k+1$ we have

$$
\left\langle X^{2 k+1}\right\rangle=0,
$$

while for even $n=2 k$

$$
\left\langle X^{2 k}\right\rangle=(-1)^{k}\left[\left(-\frac{1}{4}\right)\left(\mu^{2}+\nu^{2}\right)\right]^{2 k} \frac{2 k !}{k !} .
$$

The other example is the coherent-state tomogram. It is the Gaussian of the form

$$
w_{\alpha}(X, \mu, \nu)=\frac{1}{\sqrt{\pi\left(\mu^{2}+\nu^{2}\right)}} e^{-[X-\langle X\rangle]^{2} /\left(\mu^{2}+\nu^{2}\right)},
$$

where

$$
\langle X\rangle=\mu\langle q\rangle+\nu\langle p\rangle
$$

and, in view of (57), one has

$$
\langle X\rangle=\sqrt{2}\left[\mu \operatorname{Re}\left(\alpha e^{-\iota t}\right)+\nu \operatorname{Im}\left(\alpha e^{-\iota t}\right)\right]
$$


Let us discuss an analog of the first excited state of the classical harmonic oscillator. The wave function of this state reads

$$
\Psi_{1}(x)=\frac{\sqrt{2} x}{\sqrt[4]{\pi}} e^{-x^{2} / 2}
$$

The tomogram of this state can be calculated using either the Radon transform of probability density (65) or the connection of the tomogram with the wave function [6]

$$
w_{1}(X, \mu, \nu)=\frac{1}{2 \pi|\nu|}\left|\int \Psi_{1}(y) \exp \left(\frac{\iota \mu y^{2}}{2 \nu}-\frac{\iota X y}{\nu}\right) d y\right|
$$

which yields

$$
w_{1}(X, \mu, \nu)=\frac{2 X^{2}}{\left(\mu^{2}+\nu^{2}\right) \sqrt{\pi\left(\mu^{2}+\nu^{2}\right)}} e^{-X^{2} /\left(\mu^{2}+\nu^{2}\right)} .
$$

In quantum mechanics, this tomogram corresponds to the physical state. For the classical oscillator, this tomogram describes a nonphysical state with negative probability-distribution density (65). Thus, the ground-like state and the coherent state, being the Gaussian packets, describe the physical states for both classical and quantum oscillators. The wave function and tomogram of the first excited state describe the physical state for the quantum oscillator and correspond to the nonphysical state of the classical harmonic oscillator.

\section{Conclusions}

We formulate the main results of our study.

We have shown that the states of the classical harmonic oscillator can be described by the wave function and density matrix as well as by probability distributions in the phase space and tomographicprobability distributions. We have constructed the wave function of the coherent state and discussed nonphysical wave functions describing the classical analogs of excited states of the quantum harmonic oscillator. We have shown that the wave functions of the oscillator obey the same Schrödinger-like evolution equation of the quantum harmonic oscillator. Thus, there exists the formalism of describing the classical oscillator, which is equivalent to the formalism of describing the quantum harmonic oscillator. We hope to extend the quantum-like formalism applied to the classical harmonic oscillator to the case of the parametric classical oscillator (oscillator with a time-dependent frequency).

The classical parametric oscillator has time-dependent integrals of motion which are linear in position and momentum. The quantum parametric oscillator has time-dependent integrals of motion which are linear in the position and momentum operators. The integrals of motion provide the possibility to solve the Schrödinger evolution equation of the quantum parametric oscillator and to find an explicit form of the oscillator wave function. In a forthcoming publication, we will show that the analogous approach can be applied to study the evolution of the distribution function of the classical parametric oscillator by solving the kinetic Liouville equation. We will solve this equation by reducing it to the Schrödinger-like equation for the wave function of the classical parametric oscillator, in complete analogy with the case of the quantum parametric oscillator. 
We also plan to extend in a future publication the analysis of Bell inequalities based on the qubitportrait method [13] to study classical states with fluctuations. The tomographic approach to classical states provides the possibility to apply the notion of tomographic entropy to describe classical fluctuations analogously to the application of this notion in the quantum case of spin states employed in [14].

\section{Acknowledgments}

This study was partially supported by the Russian Foundation for Basic Research under Project No. 07-02-00598.

\section{References}

1. L. D. Landau and E. M. Lifshiz, Classical Mechanics [in Russian], Nauka, Moscow (1963).

2. A. S. Davidov, Quantum Mechanics [in Russian], Nauka, Moscow (1961).

3. Ya. M. Belousov and V. I. Man'ko, Density Matrix [in Russian], Moscow Physical Technical University, Moscow (2004).

4. S. Mancini, V. I. Man'ko, and P. Tombesi, Phys. Lett. A, 213, 1 (1996).

5. O. V. Man'ko, and V. I. Man'ko, J. Russ. Laser Res., 18, 407 (1997).

6. V. I. Man'ko and R. V. Mendes, Physica D, 145, 330 (2000).

7. O. V. Man'ko and V. I. Man'ko, J. Russ. Laser Res., 21, 411 (2000).

8. O. V. Man'ko, V. I. Man'ko, and O. V. Pilyavets, J. Russ. Laser Res., 26, 429 (2005).

9. I. A. Malkin and V. I. Man'ko, Dynamic Symmetries and Coherent States of Quantum Systems [in Russian], Nauka, Moscow (1979).

10. J. Radon, Ber. Sachs. Akad. Wiss., Leipzig, 69, 262 (1917).

11. E. Wigner, Phys. Rev., 40, 749 (1932).

12. N. N. Bogolubov and D. B. Shirkov, Quantum Field Theory [in Russian], Nauka, Moscow (1959).

13. V. N. Chernega and V. I. Man'ko, J. Russ. Laser Res., 28, 103 (2007).

14. V. N. Chernega, O. V. Man'ko, V. I. Man'ko, et al., J. Russ. Laser Res., 27, 132 (2006). 